\title{
PARALLEL SOLVERS COMPARISON FOR AN INVERSE PROBLEM IN FRACTIONAL CALCULUS
}

\author{
Pasquale De Luca ${ }^{1}$, Ardelio Galletti ${ }^{2}$ and Livia Marcellino ${ }^{2}$ \\ ${ }^{1}$ Department of Computer Science, University of Salerno, Fisciano, Italy \\ ${ }^{2}$ Department of Science and Technology, University of Naples Parthenope, Napoli, Italy
}

\begin{abstract}
High-Performance Computing (HPC) is a fundamental tool for improving the performance of many algorithms in terms of time, especially for large-scale problems. In the last years, various HPC architectures have been developed to quickly process data in many research areas and at the same time the HPC tools has become very important. In addition, the development of scientific libraries for parallel computing plays a key role in achieving better performance. In particular, thanks to the computational power of Graphic Processing Units, the most popular and inexpensive accelerators, the parallel computing field has become almost a standard process for data management. Hence, the porting of many standard numerical libraries on these architectures produced excellent results. In this work, we deal with a two-dimensional time fractional diffusion problem. More in detail, we analyze the performance of some parallel codes, specifically designed to solve it, implemented in different architectures. Moreover, a further GPU version is proposed and compared with the above implementations.
\end{abstract}

\section{KEYWORDS}

Parallel Algorithms, Multicore, GPU Computing, CUDA, Fractional Calculus

\section{INTRODUCTION}

The data size to be processed has been growing exponentially in the last years in the digital era. Therefore, the computational time and memory requirements, which characterize large problems, have been increased. The parallel computing approach represents the main source of speed improvement. In fact, there are many application fields, such as Mathematical Modeling, Computational Fluid Dynamics, Numerical Analysis etc., which benefit from the use of parallelization techniques. So, an accurate performance analysis certainly plays a key role. Clearly, the performance of a parallel algorithm depends on both the algorithms and the architectures used. Moreover, each architecture has a specific role for each problem and this is the major cause and the greatest motivation of the hardware evolution. For many decades the development of HPC technologies has focused on the need to work with machines with more processing units, in order to obtain the best performance. Today, thanks to modern multicore and Graphic Processing Units (GPUs) architectures, many problems can be investigated and the analysis of related performance, in terms of accuracy and efficiency, is possible as well as mandatory. Moreover, the rapid increase in the performance of graphics hardware and software has made GPUs, and related parallel numerical libraries, strong candidates for performing many computing intensive tasks.

This paper aims to provide a comparison analysis of some parallel codes, in terms of performance. These codes have been developed to solve a numerical problem in fractional calculus. Briefly, the theory of fractional calculus has become more and more central in several application fields, both in engineering and science. Indeed, inherent features of several chemistry, mechanics, and physics processes are potently described by means of mathematical fractional models (Mohebbi et al. 2013, Piret et al. 2013, Aslefallah et al. 2015). Here, we are focused on a two-dimensional inverse Caputo time fractional diffusion equation. The numerical procedure, we consider, is based on the discretization of the Caputo fractional derivative and on the use of a meshless localized collocation method exploiting the radial basis functions (RBFs) properties. In fact, nowadays the use of RBFs in fractional calculus is a well established procedure (Fasshauer 2007, Cuomo et al. 2017). This is mainly because they do not require the mesh construction, which is a well known too expensive 
task. The numerical scheme we deal with has been previously parallelized both in multicore and GPU architectures (De Luca et al. 2020, De Luca et al. 2020). Those implementations have been proven to be very efficient, but no comparison to find the best performance, in terms of execution time, has been discussed. Hence, here the objective is first of all to provide a clear comparison between the two versions. Then, we will exploit the potentiality of the GPU algorithm, for investigating the use of other library solvers to tackle the main computational kernel which consists in the numerical solution of a large and sparse linear system of equations. More precisely, starting by the previous GPU-parallel version of our software in (De Luca et al. 2020, De Luca et al. 2020) based on ad hoc parallel CUDA-kernels and efficient usage of parallel numerical libraries available on CUDA (Compute Unified Device Architecture) environment, we propose a further solver and measure the effects in terms of execution times and accuracy. Tests show that the new version can overcome in accuracy the other ones.

The rest of the paper is organized as follows: in section 2 the numerical problem and the related sequential algorithm are recalled; section 3 deals with the description of the main details of both parallel implementations, multicore and GPUs, of the algorithm. The use of different solvers is also a contribution in this section; in section 4 we provide tests and experiments to analyze and compare our codes in terms of performance and reliability; finally, in section 5 conclusions close the paper.

\section{FRACTIONAL MODEL AND ALGORITHM}

The starting model is the two-dimensional inverse time fractional diffusion equation $[6,7,8]$, defined as follows:

$$
{ }_{0}^{c} D_{t}^{\alpha} v(x, t)=\kappa \Delta v(x, t)+f(x, t),
$$

where: $\boldsymbol{x}=(x, y) \in \boldsymbol{\Omega} \subseteq \boldsymbol{R}^{2}$ and $\left.\left.t \in\right] 0, T\right]$. Here, the expression: $\quad{ }_{0}^{c} \boldsymbol{D}_{t}^{\alpha}=\frac{\partial^{\alpha}}{\partial \boldsymbol{t}^{\alpha}}$ denotes the Caputo fractional derivative (Cuomo et al. 2016, Podlubny 1999). The order $\alpha$ is in ]0,1]. The model also includes following initial and Dirichlet boundary conditions:

$$
\begin{gathered}
v(x, 0)=\varphi(x), x \in \Omega, \\
\left.\left.v(x, t)=\psi_{1}(x, t), x \in \Gamma_{1}, t \in\right] 0, T\right], \\
\left.\left.v(x, t)=\psi_{2}(x) \rho(t), x \in \Gamma_{2}, t \in\right] 0, T\right],
\end{gathered}
$$

and the solutions sought $v(\boldsymbol{x}, t)$ and $\rho(t)$ are unknown functions that verify the additional non-local boundary condition:

$$
\left.\left.\iint_{\Omega} v(x, t) d x=h(t), t \in\right] 0, T\right]
$$

\subsection{Numerical Model}

Following the time discretization described in (De Luca et al. 2020), let: $\tau>0$ be a time step and set: $t^{n}=n \tau$ for $n=0, \ldots, T / \tau$

By putting $t=t^{n+1}$ in (1), we get:

$$
{ }_{0}^{c} D_{t}^{\alpha} v\left(x, t^{n+1}\right)=\kappa \Delta v\left(x, t^{n+1}\right)+f\left(x, t^{n+1}\right),
$$

where: $\left(\boldsymbol{x}, t^{n+1}\right) \in \Omega \times(0, T]$.

Then, by using the second-order time discretization (Tian et al. 2015, Kibas et al. 2006), we obtain:

$$
\left.{ }_{0}^{c} D_{t}^{\alpha} v(x, t)\right]_{t=t^{n+1}}=\sum_{j=0}^{n+1} \frac{\omega^{\alpha}(j)}{\tau^{\alpha}} v\left(x, t^{n+1-j}\right)-\frac{t^{-\alpha}}{\Gamma(1-\alpha)} v(x, 0)+O\left(\tau^{2}\right)
$$

where the parameters are: 
$\boldsymbol{\omega}^{\alpha}(\boldsymbol{j})=\left\{\begin{array}{cc}\frac{\alpha+2}{2} p_{0}^{\alpha}, & \boldsymbol{j}=\mathbf{0}, \\ \frac{\alpha+2}{2} p_{j}^{\alpha}-\frac{\alpha}{2} p_{j-1}^{\alpha}, & j>\mathbf{0},\end{array} \quad p_{j}^{\alpha}=\left\{\begin{array}{cl}1, & j=0, \\ \left(1-\frac{\alpha+1}{j}\right) p_{j-1}^{\alpha}, & j \geq 1 .\end{array}\right.\right.$

Finally, combining equations (4) and (5), we have following discrete time model:

$$
\frac{\omega^{\alpha}(0)}{\tau^{\alpha}} v^{n+1}-\kappa \Delta v^{n+1}=-\sum_{j=1}^{n} \frac{\omega^{\alpha}(j)}{\tau^{\alpha}} v^{n+1-j}+\frac{t^{-\alpha}}{\Gamma(1-\alpha)} v^{0}+f^{n+1}
$$

where for $j=0, \ldots, n+1$ we set $\boldsymbol{f}^{n+1}=\boldsymbol{f}\left(\boldsymbol{x}, \boldsymbol{t}^{n+1}\right)$ and $\boldsymbol{v}^{n+1-j}=\boldsymbol{v}\left(\boldsymbol{x}, \boldsymbol{t}^{n+1-j}\right)$.

Now, to get a discretized solution we use a meshless localized collocation method. More in detail, we partition the global domain $\Omega$ into local sub-domains $\Omega_{i}(i=1, \ldots, N)$, related to every point, that cover $\Omega$. So, we define radial point interpolation shape functions, $\phi_{i}$ for all $\Omega_{i}$, each of which corresponds to a unique local field point $\boldsymbol{x}_{i}$. The chosen RBFs are the generalized multiquadric radial basis function (GMQ-RBF): $\phi(\boldsymbol{r})=\left(\boldsymbol{r}^{2}+\boldsymbol{c}^{2}\right)^{q}$

where $C$ is the shape parameter and $q=2.5$ is fixed. Assuming that $v$ can be approximated by:

$$
v(x)=\sum_{i=1}^{N} \phi_{i}(x) v_{i}
$$

where the norm $\boldsymbol{\phi}_{\boldsymbol{i}}(\boldsymbol{x})=\boldsymbol{\phi}\left(\left\|\boldsymbol{x}-\boldsymbol{x}_{\boldsymbol{i}}\right\|_{2}\right)$ denotes the classical Euclidean distance. Finally, by using expansion (7) in (2), (3) and (6), the equations of the discrete model are obtained:

$$
\begin{gathered}
\sum_{i=1}^{N}\left[\frac{\boldsymbol{\omega}^{\alpha}(\mathbf{0})}{\boldsymbol{\tau}^{\alpha}} \boldsymbol{\phi}_{i}\left(\boldsymbol{x}_{\boldsymbol{j}}\right)-\boldsymbol{\kappa}\left[\frac{\boldsymbol{\partial}^{2} \boldsymbol{\phi}_{\boldsymbol{i}}}{\boldsymbol{\partial} \boldsymbol{x}^{\mathbf{2}}}+\frac{\boldsymbol{\partial}^{2} \boldsymbol{\phi}_{\boldsymbol{i}}}{\boldsymbol{\partial} \boldsymbol{y}^{2}}\right]\left(\boldsymbol{x}_{\boldsymbol{j}}\right)\right] \boldsymbol{v}_{\boldsymbol{i}}^{n+1}= \\
-\sum_{j=1}^{n} \frac{\omega^{\alpha}(j)}{\tau^{\alpha}} \sum_{i=1}^{N} \phi_{i}\left(\boldsymbol{x}_{j}\right) v_{i}^{n+1-j}+\frac{t^{-\alpha}}{\Gamma(1-\alpha)} \sum_{i=1}^{N} \phi_{i}\left(\boldsymbol{x}_{j}\right) v^{0}+f^{n+1},\left(j=1, \ldots, N_{\Omega}\right) \\
\sum_{i=1}^{N} \phi_{i}\left(\boldsymbol{x}_{j}\right) v_{i}^{n+1}=\psi_{1}^{n+1}\left(\boldsymbol{x}_{j}\right),\left(j=N_{\Omega}+1, \ldots, N_{\Omega}+N_{\Gamma_{1}}\right) \\
\sum_{i=1}^{N} \phi_{i}\left(\boldsymbol{x}_{j}\right) v_{i}^{n+1}=\psi_{2}^{n+1}\left(\boldsymbol{x}_{j}\right) \rho^{n+1},\left(j=N_{\Omega}+N_{\Gamma_{1}}+1, \ldots, N_{\Omega}+N_{\Gamma_{1}}+N_{\Gamma_{2}}\right) \\
\sum_{i=1}^{N}\left(\int_{\Omega} \phi_{i}(\boldsymbol{x}) d \Omega\right) v_{i}^{n+1}=h^{n+1} .
\end{gathered}
$$

We notice that equations (8) concern the $N_{\Omega}$ interior points in $\Omega$, while the $N_{\Gamma_{1}}$ equations (9) and the $N_{\Gamma_{2}}$ equations (10) are related to the initial and boundary conditions, respectively. Lastly, equation (11) arises from integral in (3), which is discretized with a Gauss-Legendre bidimensional quadrature rule of order 15.

\subsection{Algorithm}

The above described approach identifies a $(N+1) \times(N+1)$ linear system where the size problem depends on both boundary and interior points, that is: $N=N_{\Omega}+N_{\Gamma_{1}}+N_{\Gamma_{2}}$.

In this scenario, the discrete solution entries: $\boldsymbol{v}^{(n+1)}=\left(\boldsymbol{v}_{1}^{n+1}, \ldots, \boldsymbol{v}_{N}^{n+1}, \boldsymbol{\rho}^{n+1}\right)$ are determined by solving the linear system:

$$
\mathcal{A} v^{(n+1)}=\mathcal{B}^{(n+1)},
$$

where $\mathcal{A}$ is a $(N+1) \times(N+1)$ sparse coefficient matrix; $\mathcal{B}^{(n+1)}$ is a $(N+1)$ column vector.

We emphasize that $\mathcal{B}^{(n+1)}$, unlike $\mathcal{A}$, has to be upgraded along time steps. The sparsity of $\mathcal{A}$ arises from the local approach, that is only nearby points of each field point $\boldsymbol{x}_{i}$ are considered. Since number of neighbors can change, the $\mathcal{A}$ structure cannot be shown here explicitly. The discrete model is summarized in Algorithm 1. 


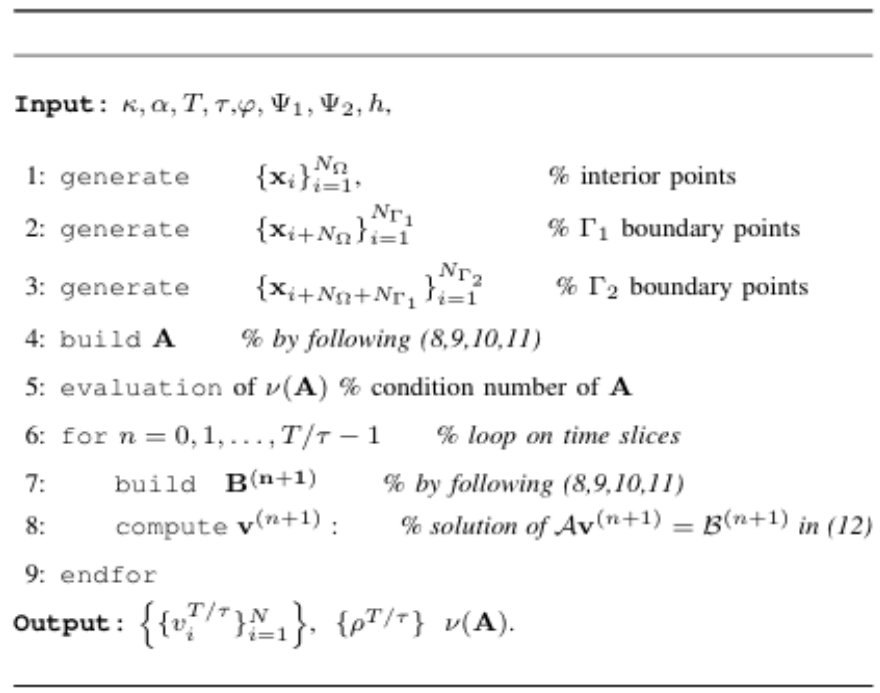

Algorithm 1. Pseudo-code

\section{PARAllel CODES}

The algorithm proposed in previous section can reach a good accuracy (De Luca et al. 2020), but executing the software on a latest-generation CPU, we observe a very large execution time. This is essentially due to the fact that, when we deal with a huge practical problem, with a large domain, the computational complexity increases considerably. Starting from these considerations we have implemented two different parallel versions of Algorithm 1: in the first one we developed a parallel algorithm specifically designed for multicore architectures and based on the Pthreads library ${ }^{1}$, while in the second one, we implemented a GPU software based on ad hoc parallel CUDA-kernels and the efficient usage of numerical libraries available for GPUs ${ }^{2}$. Here, we want to compare these two implementations and to propose a variant of the second version, which allows us to investigate the possibility of using a different solver for the main computational task.

\subsection{Multicore Parallel Code Details}

A first attempt of parallelization strategy, presented in (De Luca et al. 2020) focused on employment of multicore processors, which are widely used across many application domains. The parallel approach is based on a functional decomposition combined with a classical domain decomposition. More specifically, a pool of threads works exploiting an asynchronous parallelism by solving different tasks of the overall work in a parallel way. In particular, lines 1-4 perform the domain decomposition strategy; while a combination of domain decomposition and functional decomposition approach is used to perform in parallel lines 5-9. This combination is made as follows: the condition number of the sparse matrix is computed by a single thread, and concurrently, other threads build the right-hand side vectors (using a classical domain decomposition) and solve the sparse linear system, for each time step. Sparse linear systems are solved by using a specific routine of the CSPARSE library ${ }^{3}$. Note that, well-suited semaphores ${ }^{4}$ help the execution for critical regions, protecting the routines which are not thread-safe. This ensures a correct data consistency.

\footnotetext{
${ }^{1}$ https://computing.llnl.gov/tutorials/pthreads/

${ }^{2}$ https://docs.nvidia.com/cuda/cuda-c-programming-guide/index.html

${ }^{3}$ http://faculty.cse.tamu.edu/davis/suitesparse.html

${ }^{4}$ https://pubs.opengroup.org/onlinepubs/7908799/xsh/semaphore.h.html
} 


\subsection{GPU-Parallel Code Details: LU Version}

In (De Luca et al. 2020) another parallel approach, based on GPUs, is presented. Thanks to this last generation parallel machines, we achieve a satisfying execution of our code by using the powerful characteristics of CUDA environment, which allow us to overcome the limited memory problem, allocating the memory dynamically. In this way we reduced the transfer of host-device data. Moreover, also for this version, we combine the domain decomposition with a functional decomposition more sophisticated with respect to the multicore implementation. Therefore, following the domain decomposition-based parallelization strategy, each thread is linked at a single input point in order to perform the operations required to build the final sparse matrix (lines 1-4). Instead, to solve the main linear system, and evaluate $\boldsymbol{v}(\boldsymbol{A})$ (lines 5-9), a functional decomposition strategy is implemented. This phase makes use of a synchronization by the _ syncthreads() routines, in order to avoid any memory contention. In particular, the loop for, related to time discretization, runs in parallel on $T / \tau$ threads, to build the system and to copy of the sparse matrix in the local memory using the compressed sparse row format $\mathrm{CSR}^{5}$. Finally, the main sparse linear system is solved from the host by cusolverSpDcsrlsvlu() routine of the cuSOLVER library ${ }^{6}$.

\subsection{Another GPU Implementation: QR Version}

The results obtained in the two previous parallel versions seemed to us satisfactory, especially from a performance point of view, but also as regards the accuracy of the results. However, the sparse linear system preserves the ill conditioning problem. Therefore, in order to investigate about an accuracy improvement, here, we analyze the results which can be obtained by modifying the solver choice. More precisely, in line 8 we use the cusolverSpDcsrlsvqr() routine, instead of the cusolverSpDcsrlsvlu() previously used in the LU version. The cusolverSpDcsrlsvqr() routine implements the sparse QR factorization. Also for this routine it is necessary to store the coefficient matrix in CSR format and the functional decomposition strategy has slightly modified to ensure data consistency and to manage efficient storage of the new data structures used. This highlights the potential of our software which is written in modular blocks that can be easily adapted to new solvers.

\section{PERFORMANCE COMPARISON}

In this section we present a behavior analysis of the three parallel versions of Algorithm 1, described in section 3. The experimental tests are carried out in order to compare the different approaches for solving the inverse time fractional problem (1). Although several parallel software are available to solve many fractional problems, currently, to our knowledge, no one exists to this specific aim. In order to investigate about possible gain of performance or accuracy, several executions with different input size parameters, are presented. The three parallel codes run on a computer machine with the following technical specifications: two CPU Intel Xeon with 6 cores, E5-2609v3, $1.9 \mathrm{Ghz}, 32 \mathrm{~GB}$ of RAM 4 channels 51Gb/s memory bandwidth; two NVIDIA GeForce GTX TITAN X, 3072 CUDA cores, 1 Ghz Core clock for core, 12 GB DDR5, 336 GBs as bandwidth.

All experiments in this section are referred to the same case study (see De Luca et al. 2020). More in detail, we set:

- $\quad$ a uniform two-dimensional grid in $\Omega=[0,1]^{2}$, with: $N=\left(1+\frac{1}{h}\right)^{2}, h$ stepsize along $x, y$ directions;

- $\quad$ boundaries: $\Gamma_{2}=\{(x, y) \mid x=1,0<y<1\}$ and $\Gamma_{1}=\partial \Omega \backslash \Gamma_{2}$;

- the source function: $f(x, t)=2\left(\frac{t^{2-\alpha}}{\Gamma(3-\alpha)}-t^{2}\right) \exp (x+y)$,

- $\quad$ initial and Dirichlet conditions: $\varphi(\boldsymbol{x})=0, \quad \psi_{1}(\boldsymbol{x}, t)=t^{2} \exp (x+y), \psi_{2}(\boldsymbol{x})=\exp (x+y)$.

\footnotetext{
${ }^{5}$ https://docs.nvidia.com/cuda/cusparse/index.html\#csr-format

${ }^{6}$ https://docs.nvidia.com/cuda/cusolver/index.html
} 
The true solutions are given by: $v(x, t)=t^{2} \exp (x+y)$ and $\rho(t)=t^{2}$.

Execution times shown are taken as averages of 10 simulations.

Test 1 - The following test deals with the performance of the different implementations of Algorithm 1. In particular, Table 1 highlights the best performance among the three proposed approaches by varying the input sizes $N$, and for $\tau=1 / 100$. Here, we report the execution times, obtained for the best configuration, both for multicore and GPU implementations, that is: the threads number for multicore version is set to 12 (one per core); the Block $\times$ threads for GPU versions is set to $1 \times 512$. These optimal configurations emerged from the analysis carried out in (De Luca et al. 2020) and (De Luca et al. 2020).

Table 1. Execution times (s) comparison: serial, multicore, GPU-LU and GPU-QR

\begin{tabular}{|c|c|c|c|c|}
\hline \multicolumn{2}{|c|}{} & \multicolumn{3}{|c|}{ Parallel Time } \\
\hline $\mathbf{N}$ & Serial Time & MULTICORE & GPU-LU & GPU-QR \\
\hline $3.6 \times 10^{3}$ & $1.6 \times 10^{3}$ & 1.99 & 1.40 & 2.38 \\
\hline $8.1 \times 10^{3}$ & $1.2 \times 10^{4}$ & 7.98 & 1.86 & 2.84 \\
\hline $1.0 \times 10^{4}$ & $3.19 \times 10^{4}$ & 11.01 & 3.60 & 5.47 \\
\hline $1.69 \times 10^{4}$ & $1.66 \times 10^{5}$ & 29.01 & 5.20 & 7.53 \\
\hline $1.98 \times 10^{4}$ & $2.46 \times 10^{5}$ & 38.80 & 8.12 & 11.48 \\
\hline $2.25 \times 10^{4}$ & $3.9 \times 10^{5}$ & 68.01 & 8.12 & 11.95 \\
\hline
\end{tabular}

An very appreciable improving of performance, with respect to serial execution times, has already obtained by the multicore version. Better results, in terms of execution times, are provided by the GPU versions. Such an acceleration level mainly depends on the higher optimization of the parallel versions compared to the sequential one. Finally, we observe that the GPU-LU version returns the best gain of efficiency, regardless of the size problem. We highlight that the execution times in Table 1 refer to the whole computational process, including all phases described in Algorithm 1. Conversely, next experiment concerns the performance analysis, for the GPU codes, of just a portion of the process, that is the sparse linear system task.

Test 2 - Here, a specific comparison between GPU versions is presented. Since, the two versions mainly differ from each other for the routine used for solving the main task, i.e. the sparse linear system, we just limit out attention to their behaviour. We recall that GPU-LU version uses the routine cusolverSpDcsrlsvlu, which is based on the sparse LU factorization, while the GPU-QR version employs the routine cusolverSpDcsrlsvqr, based on the sparse QR factorization. The experiments, shown in Table 2, include several values of $\boldsymbol{N}$ and $\boldsymbol{\tau}$ and exploit the best configuration Block ltimes thread $=\mathbf{1} \times \mathbf{5 1 2}$.

Table 2. GPU solvers execution times (in milliseconds) cusolverSpDcsrlsvlu(LU) vs cusolverSpDcsrlsvqr(QR)

\begin{tabular}{|c|c|c|c|c|c|c|}
\hline $\mathbf{N}$ & solver & $\tau=\frac{1}{10}$ & $\tau=\frac{1}{20}$ & $\tau=\frac{1}{40}$ & $\tau=\frac{1}{80}$ & $\tau=\frac{1}{160}$ \\
\hline \multirow{2}{*}{$20^{2}$} & LU & 71.12 & 100.81 & 137.37 & 212.88 & 387.68 \\
\cline { 2 - 7 } & QR & 93.23 & 153.24 & 221.93 & 322.09 & 540.15 \\
\hline \multirow{2}{*}{$40^{2}$} & LU & 83.12 & 180.82 & 180.82 & 271.96 & 488.67 \\
\cline { 2 - 7 } & QR & 94.58 & 133.07 & 229.39 & 368.60 & 592.78 \\
\hline \multirow{2}{*}{$60^{2}$} & LU & 108.47 & 156.92 & 230.32 & 329.03 & 460.15 \\
\cline { 2 - 7 } & QR & 153.23 & 247.86 & 364.96 & 506.71 & 703.76 \\
\hline \multirow{2}{*}{$80^{2}$} & LU & 132.88 & 198.70 & 279.86 & 412.81 & 586.94 \\
\cline { 2 - 7 } & QR & 195.73 & 294.35 & 392.18 & 576.19 & 848.12 \\
\hline \multirow{2}{*}{$100^{2}$} & LU & 150.31 & 268.95 & 379.55 & 489.77 & 823.36 \\
\cline { 2 - 7 } & QR & 263.15 & 342.99 & 518.57 & 742.91 & 1096.57 \\
\hline
\end{tabular}

Experimental results emphasize that the $\mathrm{QR}$ solver requires more time with respect to $\mathrm{LU}$ solver, also when a small input size is given. In detail, LU routine is about 1.5 times faster than the QR routine. Moreover, looking at execution times in Table 2, separately for LU and QR versions, values monotonically increase both with the size problem $\boldsymbol{N}$ and the number of time steps $\boldsymbol{T} / \boldsymbol{\tau}$, in agree with the expected computational cost. Despite the different execution times both versions offer similar behaviour regard the provided accuracy. 
Test 3 - Here, we are interested in measuring the effect of using the two GPU versions, in terms of accuracy. The accuracy is computed as the error, due to the numerical approximation introduced by using both time discretization and the local collocation approach, that is the error defined as the RMSE between $v$ and its computed value $\tilde{\boldsymbol{v}}$. Table 3 highlights the accuracy provided for $\mathrm{N}=10^{\wedge} 4$ and by varying the time step $\tau$ between from $1 / 10$ to $1 / 160$.

Table 3. Accuracy for several executions by using different decomposition approaches, varying time stepping

\begin{tabular}{|c|c|c|c|c|c|}
\hline version & $\tau=\frac{1}{10}$ & $\tau=\frac{1}{20}$ & $\tau=\frac{1}{40}$ & $\tau=\frac{1}{80}$ & $\tau=\frac{1}{160}$ \\
\hline GPU-LU & $\mathbf{5 . 5 0 e - 0 5}$ & $\mathbf{3 . 5 4 e - 0 5}$ & $2.95 \mathrm{e}-05$ & $\mathbf{2 . 7 5 e - 0 5}$ & $2.64 \mathrm{e}-05$ \\
\hline GPU-QR & $3.63 \mathrm{e}-04$ & $9.94 \mathrm{e}-05$ & $\mathbf{2 . 8 0 e - 0 5}$ & $2.85 \mathrm{e}-05$ & $\mathbf{2 . 1 7 e - 0 5}$ \\
\hline
\end{tabular}

The results in Table 3 show that both versions can offer the same level of accuracy, regardless the time step value. Moreover, the best results (in bold) are alternatively provided from the two codes. So, we can conclude that both implementations, despite the execution times required, can be used for an accurate computation. We point out that previous conclusions do not change as the number of centers $\boldsymbol{N}$ varies.

\section{CONCLUSION}

In this paper, we analyzed the performance of three parallel codes, specifically designed for solving a two-dimensional time fractional diffusion problem. We started from two previously implemented versions of the parallel algorithm: one on multicore architecture and the other in a GPU environment. Then, we also provided a new GPU version, based on QR factorization, and compared all parallel algorithms in terms of performance and accuracy. Tests showed that the proposed new version can offer better accuracy results.

\section{ACKNOWLEDGEMENT}

This paper has been supported by project Algoritmi innovativi per interpolazione, approssimazione $e$ quadratura (AIIAQ) and project Algoritmi numerici e software per il trattamento di dati su larga scala in ambienti HPC (LSDAHPC)

\section{REFERENCES}

Abbasbandy, et al. Comparison of meshless local weak and strong forms based on particular solutions for a non-classical 2-D diffusion model, Engineering Analysis with Boundary Elements, 39 (2014) 121-128.

Aslefallah, Mohammad et al. "Nonlinear fractional integro-differential reaction-diffusion equation via eadial basis functions." The European Physical Journal Plus 130.3 (2015): 47.

Cuomo, et al. "A GPU-Parallel Algorithm for ECG Signal Denoising Based on the NLM Method," 2016 30th International Conference on Advanced Information Networking and Applications Workshops (WAINA), Crans-Montana, 2016, pp. 35-39.

Cuomo, S., et al. Reconstruction of implicit curves and surfaces via RBF interpolation (2017) Applied Numerical Mathematics, 116, pp. 157-171. DOI: 10.1016/j.apnum.2016.10.016

D’Amore, L. et al. (2011, September). A Parallel Three-dimensional Variational Data Assimilation Scheme. In AIP Conference Proceedings (Vol. 1389, No. 1, pp. 1829-1831). American Institute of Physics.

De Luca P., et al. (2020) Performance Analysis of a Multicore Implementation for Solving a Two-Dimensional Inverse Anomalous Diffusion Problem. In: Sergeyev Y., Kvasov D. (eds) Numerical Computations: Theory and Algorithms. NUMTA 2019. Lecture Notes in Computer Science, vol 11973. Springer, Cham

De Luca, P., et al. (2020) A gpu-cuda framework for solving a two-dimensional inverse anomalous diffusion problem. In: Foster, I., Joubert, G.R., Ku`cera, L., Nagel, W.E., Peters, F. (eds) Parallel Computing: Technology Trends, Advances in Parallel Computing. Vol 36. pp 311 - 320. IOS Press, 2020.

Fasshauer, Gregory E. Meshfree approximation methods with MATLAB. Vol. 6. World Scientific, 2007. 
Kilbas, et al. Theory and Application of Fractional Differential Equations, North Holland Mathematics Studies, 204 (2006). Mohebbi, Akbar, et al. "The use of a meshless technique based on collocation and radial basis functions for solving the time fractional nonlinear Schrodinger equation arising in quantum mechanics." Engineering Analysis with Boundary Elements 37.2 (2013): 475-485.

Piret, CeCile et al.. "A radial basis functions method for fractional diffusion equations.” Journal of Computational Physics 238 (2013): 71-81.

Podlubny, Fractional Differential Equation, Academic Press, 1999.

Q. Liu, et al. An implicit RBF meshless approach for time fractional diffusion equations, Comput Mech, 48 (2011) 1-12.

Tian et al. A class of second order difference approximations for solving space fractional diffusion equations, Mathematics of Computation, 84 (2015) 1703-1727.

Yan, et al. The method of approximate particular solutions for the time-fractional diffusion equation with a non-local boundary condition, Computers and Mathematics with Applications, 70 (2015) 254-264. 